\title{
Maturation of protective immunity induced by SIV $\Delta$ nef correlates with differential expression of transcription factors in SIV-specific CD8+ T cells
}

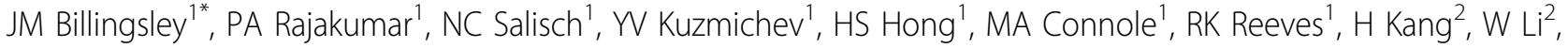 \\ RP Johnson ${ }^{1}$
}

From AIDS Vaccine 2012

Boston, MA, USA. 9-12 September 2012

\section{Background}

Protective immunity against vaginal challenge in SIVAnef-vaccinated macaques develops at 20 weeks after vaccination, whereas the magnitude of SIV-specific CD8+ $\mathrm{T}$ cell responses peaks at 5 weeks. SIV-specific CD8+ $\mathrm{T}$ cells phenotypically mature from week 5 to 20 , as characterized by upregulation of CCR7 and CD127, suggesting that the quality of the CD8 $+\mathrm{T}$ cell response may correlate with protection.

\section{Methods}

Highly parallel qRT-PCR was used to characterize the expression of 21 transcription factors (TFs) in T cells sorted into naïve, central, transitional, and effector memory subsets, and in SIV Gag CM9 and Tat SL8-specific CD8 $+\mathrm{T}$ cells obtained at wk5 and wk20 after SIV239 $\Delta$ nef vaccination.

\section{Results}

Unsupervised clustering organized $\mathrm{T}$ cell samples into groups concordant with cell surface phenotype. SIV-specific CD8+ cells segregated into wk5 and wk20 clusters. 11 of $21 \mathrm{TFs}$ were expressed at significantly different levels at wk20 than at wk5. Wk20 cells exhibited increased levels of TFs associated with both quiescence and maintenance of effector function. Furthermore, 7 TFs were significantly differentially expressed between SIV Gag and SIV Tat-specific wk20 populations. Principal component analysis suggests the Gag-specific cells may be more effector-like and the Tat-specific cells more transitional or central memorylike.

\section{Conclusion}

Our data indicate distinct transcriptional profiles of different memory $\mathrm{T}$ cell subsets and clear differences between wk5 and wk20 SIV-specific CD8+ T cell transcriptomes. The mature wk $20 \mathrm{CD} 8+\mathrm{T}$ cell response temporally correlated with protection is characterized by the expression of transcription factors associated with both central memory and effector memory $\mathrm{T}$ cells. Additionally, wk20 Gag-specific cells exhibit a more effector-like expression profile than Tat-specific cells, which is consistent with the Tat epitope exhibiting more rapid CTL escape kinetics than the Gag epitope. Analysis of transcription factor expression therefore provides a valuable complement to the analysis of memory cell differentiation based on classical phenotypic markers.

\section{Author details}

${ }^{1}$ New England Primate Research Center Harvard Medical School, Southborough, MA, USA. University of Massachusetts Medical School, Worcester, MA, USA.

Published: 13 September 2012

doi:10.1186/1742-4690-9-S2-P33

Cite this article as: Billingsley et al:: Maturation of protective immunity induced by SIV $\Delta$ nef correlates with differential expression of transcription factors in SIV-specific CD8+ T cells. Retrovirology 2012 9(Suppl 2):P33.

'New England Primate Research Center Harvard Medical School,

Southborough, MA, USA

Full list of author information is available at the end of the article

(c) 2012 Billingsley et al; licensee BioMed Central Ltd. This is an Open Access article distributed under the terms of the Creative 